\title{
Short-term dietary restriction ameliorates brain injury after cardiac arrest by modulation of mitochondrial biogenesis and energy metabolism in rats
}

\author{
Minggen Zhou ${ }^{1,2 \#}$, Tao Yu ${ }^{2,3}$, Xiangshao Fang ${ }^{2,3}$, Qiulin $\mathrm{Ge}^{2,3}$, Fengqing Song ${ }^{2,3}$, Zitong Huang ${ }^{2,3}$, \\ Longyuan Jiang ${ }^{2,3}$, Peng Wang ${ }^{2,3}$
}

${ }^{1}$ Department of Critical Care Medicine, Sun Yat-sen Memorial Hospital, Sun Yat-sen University, Guangzhou, China; ${ }^{2}$ Department of Emergency Medicine, Sun Yat-sen Memorial Hospital, Sun Yat-sen University, Guangzhou, China; ${ }^{3}$ Institute of Cardiopulmonary Cerebral Resuscitation, Sun Yat-sen University, Guangzhou, China

Contributions: (I) Conception and design: L Jiang, P Wang; (II) Administrative support: None; (III) Provision of study materials or patients: All authors; (IV) Collection and assembly of data: M Zhou, T Yu; (V) Data analysis and interpretation: All authors; (VI) Manuscript writing: All authors; (VII) Final approval of manuscript: All authors.

"These authors contributed equally to this work.

Correspondence to: Peng Wang, PhD; Longyuan Jiang, MD. Sun Yat-sen Memorial Hospital, Sun Yat-sen University, No. 107 Yan Jiang Xi Road, Guangzhou 510120, China. Email: wangp49@mail.sysu.edu.cn; jiangly@mail.sysu.edu.cn.

Background: Dietary restriction (DR) is a well-known intervention that increases lifespan and resistance to multiple forms of acute stress, including ischemia reperfusion injury. However, the effect of DR on neurological injury after cardiac arrest (CA) remains unknown.

Methods: The effect of short-term DR (one week of $70 \%$ reduced daily diet) on neurological injury was investigated in rats using an asphyxial CA model. The survival curve was obtained using Kaplan-Meier survival analysis. Serum S-100 $\beta$ levels were detected by enzyme linked immunosorbent assay. Cellular apoptosis and neuronal damage were assessed by terminal deoxyribonucleotide transferase dUTP nick end labeling assay and Nissl staining. The oxidative stress was evaluated by immunohistochemical staining of 8-hydroxy-2'-deoxyguanosine (8-OHdG). Mitochondrial biogenesis was examined by electron microscopy and mitochondrial DNA copy number determination. The protein expression was detected by western blot. The reactive oxygen species (ROS) and metabolite levels were measured by corresponding test kits.

Results: Short-term DR significantly improved 3-day survival, neurologic deficit scores (NDS) and decreased serum S-100 $\beta$ levels after CA. Short-term DR also significantly attenuated cellular apoptosis, neuronal damage and oxidative stress in the brain after CA. In addition, short-term DR increased mitochondrial biogenesis as well as brain PGC-1 $\alpha$ and SIRT1 protein expression after CA. Moreover, shortterm DR increased adenosine triphosphate, $\beta$-hydroxybutyrate, acetyl-CoA levels and nicotinamide adenine dinucleotide $\left(\mathrm{NAD}^{+}\right) /$reduced form of $\mathrm{NAD}^{+}(\mathrm{NADH})$ ratios as well as decreased serum lactate levels.

Conclusions: Reduction of oxidative stress, upregulation of mitochondrial biogenesis and increase of ketone body metabolism may play a crucial role in preserving neuronal function after CA under short-term DR.

Keywords: Cardiac arrest (CA); dietary restriction (DR); cardiopulmonary resuscitation (CPR); oxidative stress; ketone body

Submitted Apr 02, 2020. Accepted for publication Sep 30, 2020.

doi: $10.21037 /$ atm-20-3075

View this article at: http://dx.doi.org/10.21037/atm-20-3075 


\section{Introduction}

Global brain ischemia occurs when blood flow to the brain is halted or drastically reduced, which is commonly caused by cardiac arrest (CA). People who suffer from CA have a low chance of survival and high-quality cardiopulmonary resuscitation $(\mathrm{CPR})$ is essential to return of spontaneous circulation (ROSC) of CA victims (1). Patients after ROSC have high morbidity and mortality rates owing to a systemic ischemia/reperfusion response involving multiple organs. However, global brain ischemia injury is the most common cause of death in CA patients $(2,3)$. Therapeutic hypothermia (also called targeted temperature management) is the only effective treatment to improve neurological outcomes in CA patients $(4,5)$. To date, no other therapies have proved to be effective in ameliorating neurological injuries after CA. Hence, alternative strategies should be developed to further improve neurological outcomes of patients after CA.

Mitochondria are complex dynamic organelles in the cell that not only produce adenosine triphosphate (ATP) for maintaining cell viability but also participate in a number of intracellular processes such as oxidative stress response, metabolic signaling pathways, and determination of cell death or survival (6). Alterations in normal mitochondrial function are increasingly being recognized as a contributing factor in the brain injury after CA (7-9). The brain is responsible for a large amount of energy consumption and is extremely sensitive to energy deficits. Mitochondrial function in brain ischemia is altered due to insufficient glucose and oxygen to the tissue. The limited availability of glucose and oxygen directly impairs mitochondrial oxidative metabolism and leads to a rapid decrease of ATP and other energy-related metabolites (10). Furthermore, mitochondria are a major site for production of reactive oxygen species (ROS) in normal cells and mainly contribute to increased oxidative stress in ischemic brain. Limitations in the ability to generate ATP and increases of oxidative stress can greatly activate the cell death pathways (10). Therefore, increasing evidence demonstrates that mitochondria are important targets for neuroprotective interventions. For example, attenuating oxidative stress, preventing mitochondrial permeability transition pore (mPTP) opening, activating mitochondrial biogenesis, or modulating energy metabolism are potential ways to attenuate brain injury after CA by ameliorating the underlying mitochondrial dysfunction (11-14). Therapeutic hypothermia has been found to be effective in attenuating neurological injury after CA (4). Emerging evidence has demonstrated that mitochondria played a key role in the mechanisms of neuroprotective effect of therapeutic hypothermia, including the inhibition of mPTP opening, reduction of mitochondrial ROS, modulation of mitochondrial dynamics and increase of energy metabolism (15-18).

Dietary restriction (DR) or calorie restriction, defined as reduced food intake without malnutrition, is a wellknown intervention that increases lifespan and decreases the incidence and severity of age-related disease in numerous model organisms (19). Dietary intervention usually consists of a $20-70 \%$ reduction in diet intake without micronutrient limitation relative to an ad libitum (AL) diet. DR has been demonstrated to effectively prevent a large number of age-related neurological disorders, including learning and memory disorders $(20,21)$. In addition to preventing age-related disease, another important property of DR is increased resistance to multiple forms of acute stress, including ischemia reperfusion injury. Reports have found that a restricted diet for 3 months to 1 year had reduced ischemic injury to the heart and brain in models of heart attack and stroke, respectively $(22,23)$. Several neuroprotective mechanisms have been found in the beneficial effects of DR, including antioxidant effects, enhancement of autophagy, reduction of inflammation, and modulation of metabolism $(19,20,24)$. Although the mechanisms by which DR takes its effects are still scarce, mitochondria, as master regulators of cellular metabolism, are believed to play an important role in the cellular adaptations that occurred with the diet $(20,24,25)$. However, the effects of DR on neurological injury after CA and involved mechanisms remain unknown.

We hypothesized that DR could attenuate neurological injury after CA by protecting mitochondrial function. Therefore, this study aims to determine the effects of DR on brain injury after CA and dissect the involved molecular mechanisms. The length of time on a restricted diet required to exert its effects is not well characterized in any disease model. Here, we examined the effects of DR on brain injury in a rat model of asphyxial CA using short period of food restriction, which was one week of $70 \%$ reduced daily food availability (defined here as short-term DR). We present the following article in accordance with the ARRIVE reporting checklist (available at http://dx.doi. org/10.21037/atm-20-3075). 
A

\begin{tabular}{|c|c|c|c|c|}
\hline SAL group & $\mathrm{AL}$ & \multicolumn{2}{|c|}{ Sham surgery } & Detection \\
\hline SDR group & DR & \multicolumn{2}{|c|}{ Sham surgery } & Detection \\
\hline CAL group & AL & CA & CPR & Detection \\
\hline CDR group & DR & CA & CPR & Detection \\
\hline
\end{tabular}

B

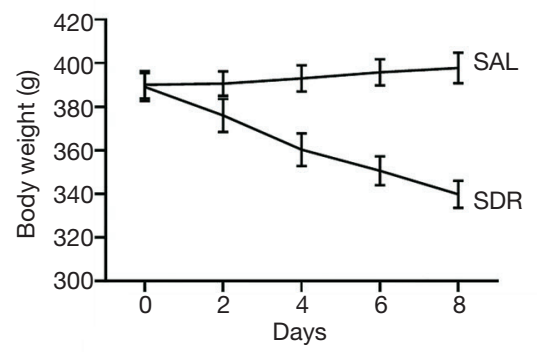

$\mathrm{E}$
C

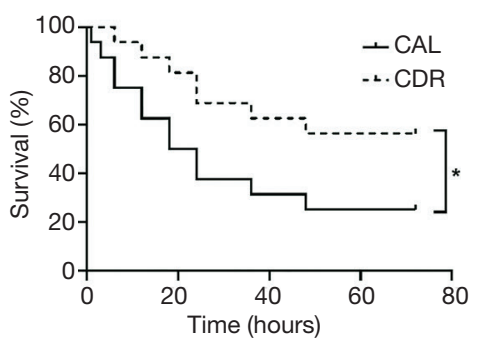

$\mathrm{D}$

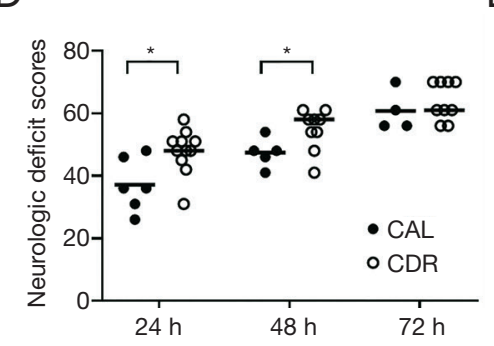

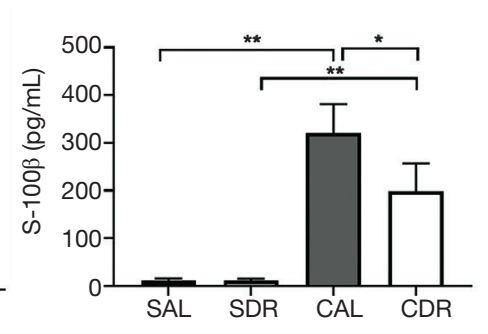

Figure 1 Short-term dietary restriction (DR) improved survival and neurological outcome of the rats after cardiac arrest (CA). (A) Schematic of experimental procedures and groups. (B) Effect of short-term DR on body weight. Data are shown as mean \pm SD. (C) Effect of shortterm DR on survival of rats during 3-day follow-up after CA. Solid line, the CAL group; dashed line, the CAL group. *, P<0.05, n=16; log rank test. (D) Effect of short-term DR on neurologic deficit scores of survived rats at 24, 48, and 72 hours after CA. Data are shown as mean $\pm \mathrm{SD},{ }^{*}, \mathrm{P}<0.05$; Kruskal-Wallis test with all pairwise multiple comparison. (E) Effect of short-term DR on serum S-100ß levels at $24 \mathrm{~h}$ after CA. Data are shown as mean $\pm \mathrm{SD},{ }^{*}, \mathrm{P}<0.05,{ }^{* *}, \mathrm{P}<0.01$; one-way ANOVA followed by Tukey test. SAL, the sham ad libitum diet control group; SDR, the sham dietary restriction control group; CAL, the ad libitum diet and CPR group; CDR, the dietary restriction and CPR group; CA, cardiac arrest; CPR, cardiopulmonary resuscitation; AL, ad libitum; DR, dietary restriction.

\section{Methods}

\section{Animals and DR}

Male Sprague-Dawley rats (380-400 g) were purchased from Experimental Animal Center of Sun Yat-sen University (Guangzhou, China). Animals were kept under standard laboratory conditions in a specific pathogen-free room at a constant temperature $\left(20-22{ }^{\circ} \mathrm{C}\right)$ with 12 hours of light/12 hours of dark exposure. All animal experiments were carried out in accordance with the National Institutes of Health Guide for the Care and Use of Laboratory Animals (8th edition; Washington DC, National Academic Press, 2011). The protocol was approved by the Institutional Animal Care and Use Committee of Sun Yatsen University (No. IACUC-DB-15-1207). Animals ( $\mathrm{n}=106)$ were randomly allocated to different treatment groups. The control rats $(\mathrm{n}=56)$ were fed an experimental diet under $\mathrm{AL}$ conditions. The DR rats $(n=50)$ were given $30 \%$ of the diet compared with the control group each day for one week. Despite significant weight loss as a result of short-term DR in rats, no morbidity or mortality was observed in the DR rats. The $\mathrm{AL}$ and $\mathrm{DR}$ rats then prepared for the CA model. An illustration of the experimental procedures and groups is provided (Figure 1A).

\section{CA model}

CA was induced by asphyxia and this model was prepared as previously described (26). Briefly, CA was induced by clamping the endotracheal tube. CA was defined as mean arterial blood pressure (MAP) $\leq 20 \mathrm{mmHg}$, which occurred approximately 4 minutes after endotracheal tube clamping. After 6 minutes of untreated CA, CPR was started by mechanical ventilation with $100 \%$ oxygen and performed precordial compression (PC). After 2 minutes of PC, epinephrine $(0.01 \mathrm{mg} / \mathrm{kg})$ was injected via the right atrium catheter. In unsuccessfully resuscitated animals, the epinephrine was repeatedly injected after 4 minutes of PC. If rats had ventricular fibrillation, defibrillation was attempted with 2-J counter shocks. The PC was stopped when there was no ROSC after 8 minutes of PC. ROSC was defined as the return of supraventricular cardiac rhythm 
with a MAP $\geq 60 \mathrm{mmHg}$ for a minimum of 5 minutes.

\section{Neurological function examination}

The neurological function of the rats at 24, 48 and 72 hours after CA was evaluated according to the method of neurologic deficit score (NDS) as previously described (27). Briefly, assessment of brain function involves the scoring of 19 items, including arousal, reflex, motor, sensory, and balance responses, resulting in scores ranging from 0 (brain death) to 80 (normal brain function). The NDSs were scored by two observers blind to group identities.

\section{Serum S-100ק levels}

Blood samples were collected from the arterial catheter at 24 hours after ROSC. The blood was allowed to clot for 30 minutes at room temperature. The clot was removed by centrifugation at $1,500 \mathrm{~g}$ for $15 \mathrm{~min}$ and the serum was stored at $-80{ }^{\circ} \mathrm{C}$ until use. Serum S-100 $\beta$ levels were detected using a rat S-100 $\beta$ enzyme-linked immunosorbent assay kit (Cusabio, China) according to the manufacturer's instructions.

\section{Nissl staining and terminal deoxyribonucleotide transferase dUTP nick end labeling (TUNEL) assay}

Rats were deeply anesthetized by intraperitoneal injection of $5 \%$ pentobarbital, and were perfused with $0.9 \%$ sodium chloride and $4 \%$ formaldehyde solution. The brain was removed immediately and stored in $4 \%$ paraformaldehyde. Following dehydration in graded xylene and ethanol, the sections were embedded in the paraffin. Embedded brain tissues were sliced into $4 \mu \mathrm{m}$ sections for staining. The Nissl staining (Beyotime, Nantong, China) was performed for detection of neuronal injury according to the manufacturer's instructions. A TUNEL assay was used to evaluate neuronal apoptosis using the in situ cell death detection kit (Roche Molecular Biochemicals, Germany). Quantitation of Nisslor TUNEL-stained neuronal cells was performed by counting the number of positive cells in seven randomly chosen fields (40x magnification) in the hippocampal CA1 area from each group.

\section{Oxidative stress analysis}

The oxidative stress in the brain at 24 hours after CA was evaluated by immunohistochemical staining of 8 -hydroxy- 2'-deoxyguanosine (8-OHdG). Brain tissues were sectioned and embedded in paraffin. The immunohistochemistry was performed as we previously described (28), and 8-OHdG antibody (diluted 1:200; Abcam, Cambridge, UK) was used. The immunostaining scores were estimated using both the percentage of positively stained cells and the staining intensity as we previously described (17). The ROS levels in the brain at 6 hours after CA were measured using the tissue ROS assay kit (Genmed Scientifics, Wilmington, DE, USA) that utilized 2',7'-dichlorofluorescein diacetate as the oxidative fluorescent probe. After the fresh brain tissues were isolated, the ROS levels were measured following the manufacturer's instructions.

\section{Western blot analysis}

For immunoblotting, PGC1- $\alpha$, SiIR1 (diluted 1:1,000; Cell Signaling Technologies, USA) and $\beta$-actin (diluted 1:2,000; Cell Signaling Technologies, USA) antibodies were used. Protein bands were detected using an enhanced chemiluminescence kit (Cell Signaling Technologies, USA). $\beta$-actin was used to normalize protein loading. The densities of protein blots were quantified by using Image J (National Institutes of Health, Bethesda, MD) and normalized to control.

\section{Mitochondrial DNA (mtDNA) copy number determination}

The total DNA (nuclear and mitochondrial) was extracted from brain tissues at 24 hours after CA by using the QIAamp DNA mini kit (Qiagen, Germany). The mtDNA copy number was measured by quantitative real time PCR as we previously described (14).

\section{Transmission electron microscopy preparation and analysis}

Animals were anesthetized and fixed by transcardiac perfusion with $4 \%$ paraformaldehyde and $2.5 \%$ glutaraldehyde in $0.1 \mathrm{M}$ phosphate buffer (PBS, pH

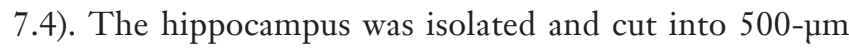
thick transverse slices which were post-fixed in the same fixative overnight and in 1\% osmium tetroxide for 1 hour. Tissue slices were then dehydrated in ascending series of ethanol and embedded in Epon. Small pieces of samples (approximate $1 \mathrm{~mm}^{2}$ in size) containing the CA1 region were cut from embedded slices and glued onto the resin. Sections were stained with uranyl acetate and lead citrate and examined in a Tecnai G2 transmission electron microscope 
(FEI, Hillsboro, OR, USA) at a final magnification of 13,500x. For counting mitochondrial numbers, seven fields of micrograph in different hippocampal CA1 neurons per animal were evaluated. Data were expressed as number per $20 \mathrm{~mm}^{2}$ (the area of neuron nucleus is not calculated).

\section{Measurement of metabolite levels}

Brain ATP levels were measured using an ATP bioluminescent assay kit (Beyotime, China) according to the manufacturer's instructions. Brain and serum $\beta$-hydroxybutyrate (BHB) concentrations were measured using a BHB colorimetric assay Kit (Biovision, Milpitas, CA, USA) according to the manufacturer's instructions. Brain acetyl-CoA levels were measured using an acetyl-CoA assay kit (Sigma-Aldrich, St. Louis, USA) according to the manufacturer's instructions. Brain nicotinamide adenine dinucleotide $\left(\mathrm{NAD}^{+}\right) /$reduced form of $\mathrm{NAD}^{+}(\mathrm{NADH})$ levels were measured using a $\mathrm{NAD}^{+} / \mathrm{NADH}$ quantification colorimetric kit (Biovision, Milpitas, CA, USA) according to the manufacturer's instructions. Serum lactate concentrations were measured by biochemistry analyzer (YSI, Yellow Springs, OH, USA).

\section{Statistical analysis}

All data were presented as mean \pm standard deviation, and analyzed using SPSS version 23.0 (IBM Corp, Armonk, NY, USA). The unpaired $t$-test was used to compare the same parameters between two groups. Normally distributed data were analyzed by one-way analysis of variance (ANOVA) followed by Tukey's post hoc test. NDSs were analyzed by the Kruskal-Wallis test with all pairwise post hoc analyses between multiple groups. The survival curve among the groups was obtained using Kaplan-Meier survival analysis and compared with a log-rank test. A two-tailed $\mathrm{P}<0.05$ was considered significant.

\section{Results}

\section{Animal physiologic data and resuscitation characteristics}

A total of 106 rats were prepared for this study. Of these rats, 56 rats were fed the AL diet and 50 rats were given DR each day for 1 week. In AL rats, 12 rats received sham operation (The SAL group), and 44 other rats underwent CPR (The CAL group) but 7 rats failed to achieve ROSC and 9 rats failed to survive in measurement time point $(6$ or 24 hours after CA). In the DR rats, 12 rats received sham operation (The SDR group), and 38 other rats underwent CPR (The CDR group) but 5 rats failed to achieve ROSC and 5 rats failed to survive in measurement time point. The rats that failed to achieve ROSC or failed to survive in measurement time point were excluded from the group. There were no significant differences in baseline physiologies, hemodynamic, and blood analytical measurements among four groups (Table 1). For animals that successfully achieved ROSC, CPR outcomes were comparative between the CAL and CDR group (Table 1).

\section{Short-term DR improved survival and neurological outcome of the rats after $C A$}

Body weight was measured to estimate the effect of shortterm DR on the rats. After a week of DR, a drastic decrease in body weight of the rats was detected $(398 \pm 7$ versus $340 \pm 6$ g, $\mathrm{P}<0.05$ ) (Figure 1B). Kaplan-Meier survival analysis showed that short-term DR significantly increased the 3-day survival time of the animals after ROSC $(\mathrm{P}<0.05)$ (Figure 1C). In addition, NDSs at 24 and 48 hours after ROSC were significantly higher in the CDR group compared with those in the CAL group $(\mathrm{P}<0.05)$ (Figure $1 D$ ). Serum S-100 $\beta$ is a prognostic marker of neurological injury and is used to predict neurological outcomes after CA. The serum S-100 $\beta$ concentration increased significantly after ROSC in the AL and DR rats $(\mathrm{P}<0.01)$. However, the serum $\mathrm{S}-100 \beta$ concentration in the CDR group was significantly decreased compared with the CAL group $(321 \pm 61$ versus $199 \pm 59 \mathrm{pg} / \mathrm{mL})(\mathrm{P}<0.05)$ (Figure 1E). These results suggest that short-term DR significantly increases the survival and neurological outcome of the rats after CA.

\section{Short-term DR protected the brain neurons from injuries after $C A$}

The Nissl staining and TUNEL assay were performed to detect the neuronal injury and apoptosis in hippocampal CA1 area at 24 hours after CA. Nissl staining showed that there were fewer Nissl bodies in the cytoplasm of neurons after ROSC in the AL and DR rats (Figure 2A). The number of viable neurons significantly decreased after ROSC in the AL and DR rats $(\mathrm{P}<0.05)$, whereas the number of viable neurons in the CDR group was significantly increased compared with the CAL group $(66 \% \pm 7 \%$ versus $83 \% \pm 5 \%, \mathrm{P}<0.01$ ) (Figure $2 B$ ). In addition, the TUNEL- 
Table 1 Baseline and CPR characteristics of all groups

\begin{tabular}{|c|c|c|c|c|}
\hline Variables & $\operatorname{SAL}(n=12)$ & $\operatorname{SDR}(n=12)$ & $\mathrm{CAL}(\mathrm{n}=44)$ & $\operatorname{CDR}(n=38)$ \\
\hline Mean artery pressure $(\mathrm{mmHg})$ & $133 \pm 7$ & $135 \pm 4$ & $136 \pm 5$ & $134 \pm 6$ \\
\hline Rectal temperature $\left({ }^{\circ} \mathrm{C}\right)$ & $36.5 \pm 0.3$ & $36.5 \pm 0.4$ & $36.9 \pm 0.4$ & $36.6 \pm 0.2$ \\
\hline $\mathrm{ETCO}_{2}(\mathrm{mmHg})$ & $33.4 \pm 3.2$ & $34.1 \pm 2.8$ & $33 \pm 3.7$ & $34.6 \pm 2.6$ \\
\hline Arterial $\mathrm{pH}$ & $7.4 \pm 0.04$ & $7.41 \pm 0.03$ & $7.44 \pm 0.04$ & $7.43 \pm 0.03$ \\
\hline CA induction time (s) & - & - & $251 \pm 25$ & $260 \pm 22$ \\
\hline Duration of PC (s) & - & - & $257 \pm 31$ & $251 \pm 36$ \\
\hline $\mathrm{CPP}$ in $\mathrm{PC} 1(\mathrm{mmHg})$ & - & - & $14 \pm 3$ & $15 \pm 2$ \\
\hline
\end{tabular}

Values are presented as mean \pm SD. There are no significant differences between the groups. One-way ANOVA followed by Tukey test for four groups and unpaired $t$-test for two groups. SAL, the sham ad libitum diet control group; SDR, the sham dietary restriction control group; CAL, the ad libitum diet and CPR group; CDR, the dietary restriction and CPR group; CPR, cardiopulmonary resuscitation group; $\mathrm{ETCO}_{2}$, end-tidal $\mathrm{CO}_{2}$; CA, cardiac arrest; $\mathrm{PC}$, precordial compression; CPP, coronary perfusion pressure; PCn indicates $\mathrm{n}$ minutes after precordial compression.

positive apoptotic cells significantly increased after ROSC in the $\mathrm{AL}$ and $\mathrm{DR}$ rats $(\mathrm{P}<0.01)$. However, the number of TUNEL-positive apoptotic cells in the CDR group was significantly lower than those in the CAL group $(24 \% \pm 4 \%$ versus $15 \% \pm 4 \%, \mathrm{P}<0.01$ ) (Figure $2 A, C$ ). These results suggest that short-term DR significantly decreases brain cell damage after CA.

\section{Short-term DR decreased brain oxidative stress after $C A$}

In nuclear and mitochondrial DNA, 8-OHdG is one of the predominant forms of free radical-induced oxidative lesions, and has therefore been widely used as a biomarker for oxidative stress. Brain oxidative stress was detected in four groups at 24 hours after $\mathrm{CA}$ by $8-\mathrm{OHdG}$ immunostaining. The results showed that there were more 8-OHdG-positive cells in hippocampal CA1 and cerebral cortex after ROSC in the AL and DR rats (Figure $3 A$ ). However, the staining scores of 8-OHdG in hippocampal CA1 and cerebral cortex in the CDR group were all significantly lower than those in the CAL group $(\mathrm{P}<0.01)$ (Figure $3 B)$. In addition, brain ROS levels were detected in four groups at 6 hours after CA. The ROS levels were significantly increased after ROSC in the $\mathrm{AL}$ and $\mathrm{DR}$ rats $(\mathrm{P}<0.01)$, whereas the ROS levels in the CDR group were significantly decreased compared with the CAL group $(3 \pm 0.5$ versus $1.9 \pm 0.3, \mathrm{P}<0.01)$ (Figure $3 C$ ). These studies suggest that short-term DR decreased brain oxidative stress and oxidative DNA damage after CA.

\section{Short-term DR increased brain mitochondrial biogenesis after $C A$}

Mitochondrial biogenesis is a cellular quality control process by which the cell reconstitutes functional mitochondria. To determine the effect of short-term DR on mitochondrial biogenesis after CA, the number of mitochondria in the brain was calculated at 24 hours after CA by electron microscopy analysis (Figure 4A). The electron microscopy analysis showed that there were more mitochondria after ROSC in the AL and DR rats $(\mathrm{P}<0.05)$, and there were more mitochondria in the CDR group than the CDL group $(19 \pm 1$ versus $14 \pm 1, \mathrm{P}<0.01)$ (Figure $4 B$ ). Furthermore, the electron microscopy analysis also showed that there were more mitochondria in the SDR group than the SDL group $(12 \pm 1$ versus $9 \pm 1, \mathrm{P}<0.05)$ (Figure $4 B$ ). In addition, the number of mitochondria in the brain was assessed by mtDNA copy number analysis. The results showed that the mtDNA levels were significantly increased after ROSC in the AL and DR rats $(\mathrm{P}<0.05)$, and the mtDNA levels in the CDR group were also significantly increased compared with the CAL group $(2.6 \pm 0.2$ versus $1.7 \pm 0.2, \mathrm{P}<0.05)$ (Figure $4 C$ ). Furthermore, the data also showed that the mtDNA levels were increased in the SDR group compared with the SDL group $(1.6 \pm 0.1$ versus $1.0 \pm 0.1, \mathrm{P}<0.01)$ 
A
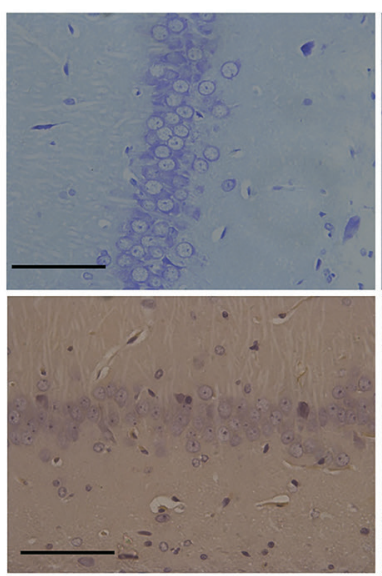

B

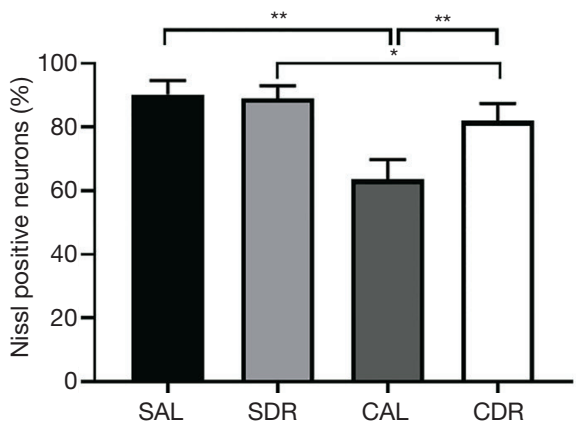

CAL
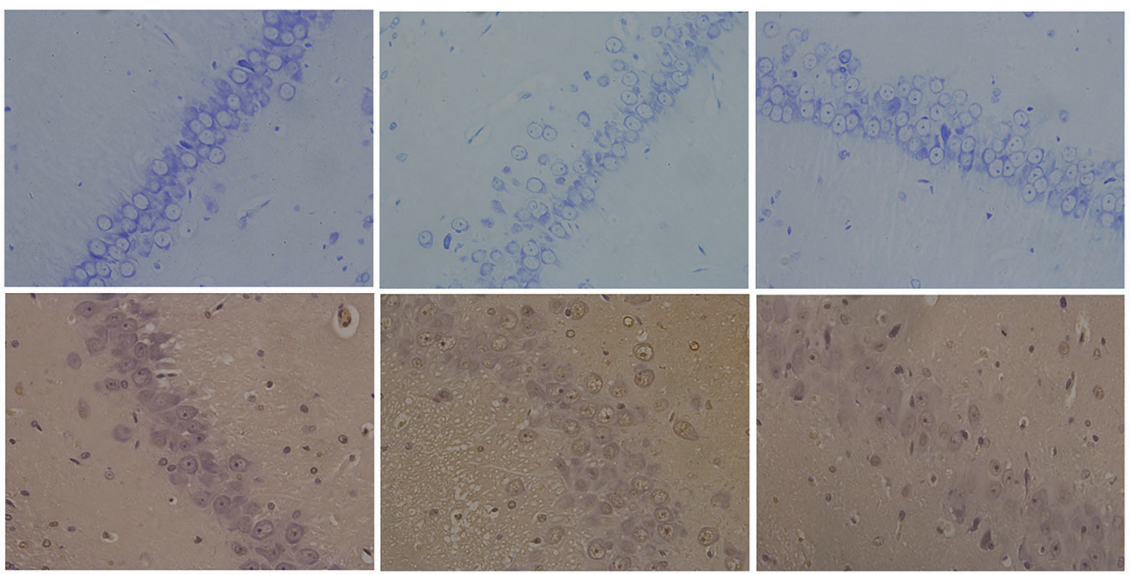

C

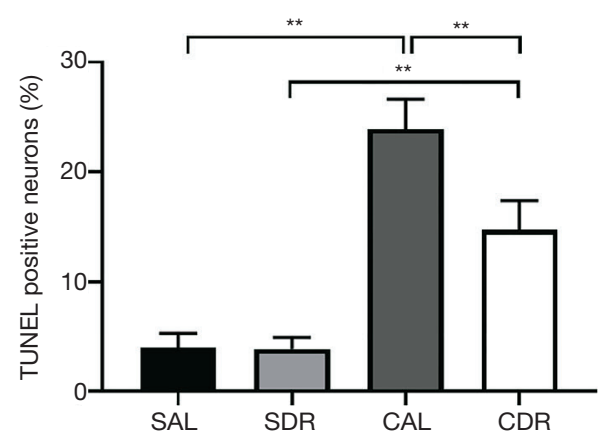

Figure 2 Short-term DR protected neurons from injury following CA of the rats. (A) Representative images of Nissl and TUNEL staining of the hippocampal CA1 neurons in four groups $(\times 400)$ at $24 \mathrm{~h}$ after CA. Top row, Nissl staining; bottom row, TUNEL staining. Scale bar $=50 \mu \mathrm{m}$. (B) Quantification of the hippocampal CA1 viable neurons by Nissl staining. (C) Quantification of the apoptotic hippocampal CA1 neurons by TUNEL staining. Data in (B) and (C) are shown as mean $\pm \mathrm{SD}, \mathrm{n}=6,{ }^{*}, \mathrm{P}<0.05$, **, $\mathrm{P}<0.01$; one-way ANOVA followed by Tukey test. SAL, the sham ad libitum diet control group; SDR, the sham dietary restriction control group; CAL, the ad libitum diet and CPR group; CDR, the dietary restriction and CPR group; TUNEL, terminal deoxynucleotide transferase-mediated dUTP-biotin nick-end labeling.

(Figure 4C). Taken together, these data demonstrate that short-term DR increases brain mitochondrial biogenesis before and after CA.

\section{Short-term DR increased brain PGC-1 $\alpha$ and SIRT1 protein expression after $C A$}

SIRT1 plays a critical role in mitochondrial biogenesis by activating PGC-1 $\alpha$ (29). The protein levels of SIRT1 and PGC- $1 \alpha$ in the brain at 24 hours after CA were assessed by western blot. The results showed that the SIRT1 and PGC$1 \alpha$ levels were significantly increased after ROSC in the AL and $\mathrm{DR}$ rats $(\mathrm{P}<0.01)$, and these protein levels in the CDR group were also significantly increased compared with the CAL group $(\mathrm{P}<0.05)$ (Figure 5). Furthermore, the data also showed that the SIRT1 and PGC-1 $\alpha$ protein levels were increased in the SDR group compared with the SDL group $(\mathrm{P}<0.05)$ (Figure 5).

\section{Ketone body involved in short-term DR mediated neurological function protection after $C A$}

The main function of mitochondria is to produce ATP, the main source of energy in all cells. The results demonstrated that the brain ATP levels were significantly decreased at 6 hours after ROSC in the AL and DR rats $(\mathrm{P}<0.01)$, whereas its levels significantly increased in the CDR group compared with the CAL group $(69 \% \pm 8 \%$ versus $85 \% \pm 8 \%$, $\mathrm{P}<0.01$ ) (Figure 6A). Ketone body represents an important alternative fuel for the cell under glucose hypometabolic 
A

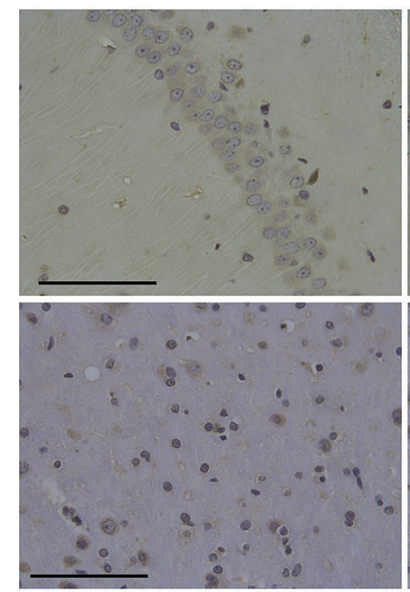

B

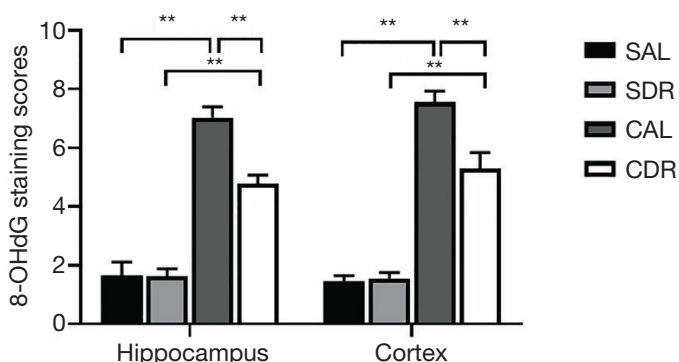

CAL
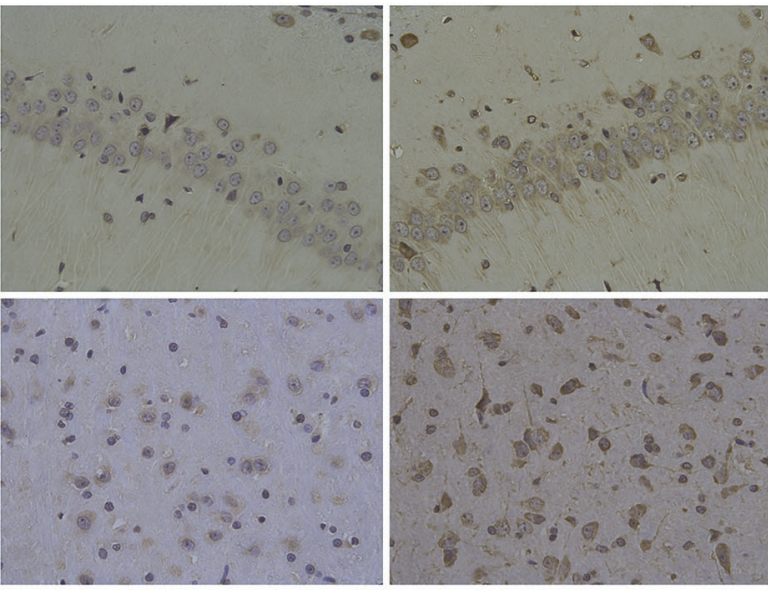

CDR

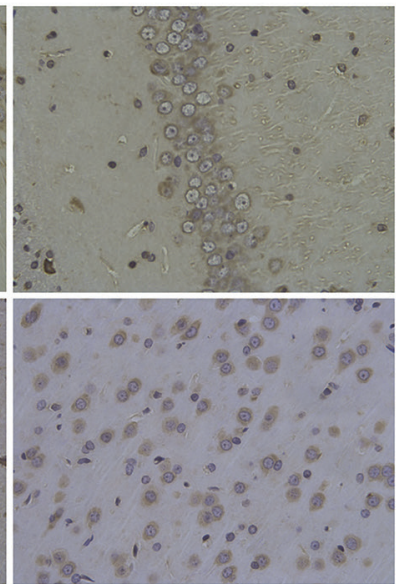

C

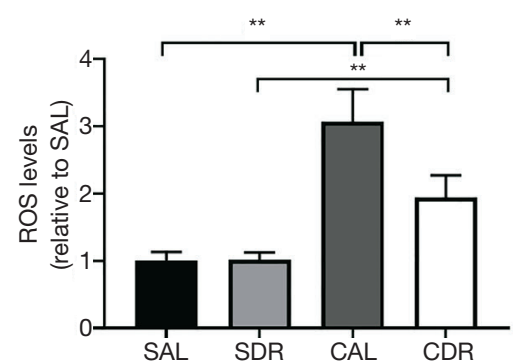

Figure 3 Short-term DR protected the brain from oxidative stress injury following CA of the rats. (A) Representative images of 8-OHdG staining of the hippocampal CA1 and cerebral cortex in four groups $(\times 400)$ at $24 \mathrm{~h}$ after CA. Top row, CA1 hippocampus; bottom row, cerebral cortex. Scale bar $=50 \mu \mathrm{m}$. (B) Quantification of 8-OHdG staining levels of hippocampal CA1 and cerebral cortex in four groups. (C) The relative ROS levels of the brain in four groups at $6 \mathrm{~h}$ after CA. Data in (B) and (C) are shown as mean $\pm \mathrm{SD}, \mathrm{n}=6,{ }^{* *}, \mathrm{P}<0.01$; one-way ANOVA followed by Tukey test. SAL, the sham ad libitum diet control group; SDR, the sham dietary restriction control group; CAL, the ad libitum and CPR group; CDR, the dietary restriction and CPR group.

conditions. To determine the role of ketone body in shortterm DR, BHB concentrations were detected in the serum and brain at 6 hours after CA. The serum and brain BHB concentrations were significantly increased in the CDR group compared with the CAL group, respectively (serum: $0.6 \pm 0.1$ versus $1.1 \pm 0.1 \mathrm{mmol} / \mathrm{L}, \mathrm{P}<0.01$; brain: $1.5 \pm 0.2$ versus $2.0 \pm 0.1 \mathrm{mmol} / \mathrm{L}, \mathrm{P}<0.01$ ) (Figure $6 B, C$ ). Acetyl$\mathrm{CoA}$ is a key metabolic signaling molecule in ATP production and can be converted from BHB. Our results showed that brain acetyl-CoA levels were significantly decreased at 6 hours after ROSC in the AL and DR rats $(\mathrm{P}<0.01)$, whereas its levels significantly increased in the CDR group compared with the CAL group $(63 \% \pm 9 \%$ versus $80 \% \pm 9 \%, \mathrm{P}<0.01$ ) (Figure $6 D$ ). Maintenance of an optimal $\mathrm{NAD}^{+} / \mathrm{NADH}$ ratio is essential for mitochondrial function (30). Our data showed that brain $\mathrm{NAD}^{+} / \mathrm{NADH}$ ratios significantly increased in the CDR group compared with the CAL group $(1.8 \pm 0.6$ versus $3.0 \pm 0.5, \mathrm{P}<0.01)$ (Figure 6E). Lactate is a metabolic end-product of anaerobic glycolysis and is a key marker of tissue injury. Our data demonstrated that the serum lactate concentrations were significantly increased at 6 hours after ROSC in the AL and $D R$ rats $(\mathrm{P}<0.01)$, whereas its levels significantly decreased in the CDR group compared with the CAL group (4.5 \pm 0.9 versus $2.5 \pm 0.6 \mathrm{mmol} / \mathrm{L}, \mathrm{P}<0.01$ ) (Figure $6 F$ ). Taken together, these data suggest that ketone body plays a critical role in short-term DR mediated neurological function protection after CA.

\section{Discussion}

The benefits of DR on aging prevention have been well 
A
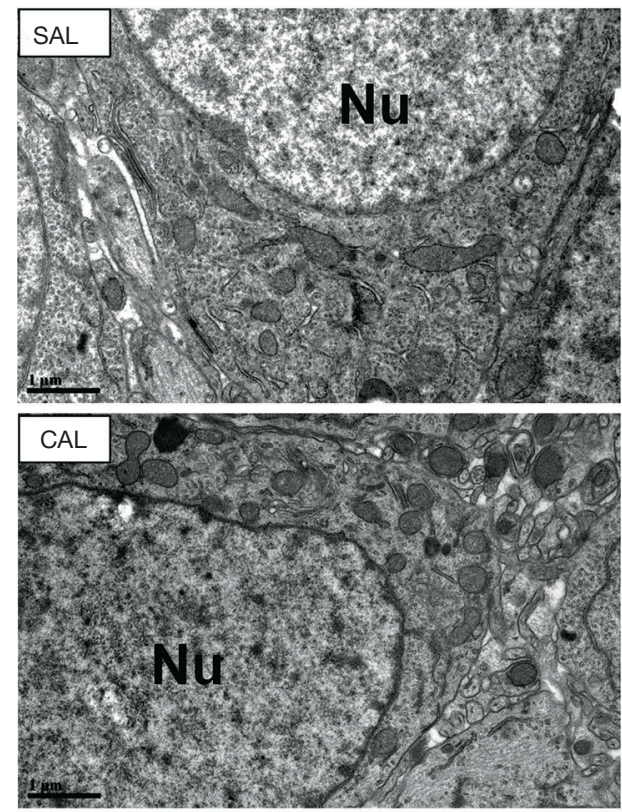

B

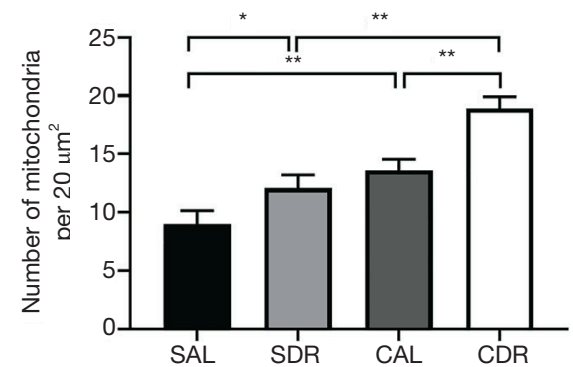

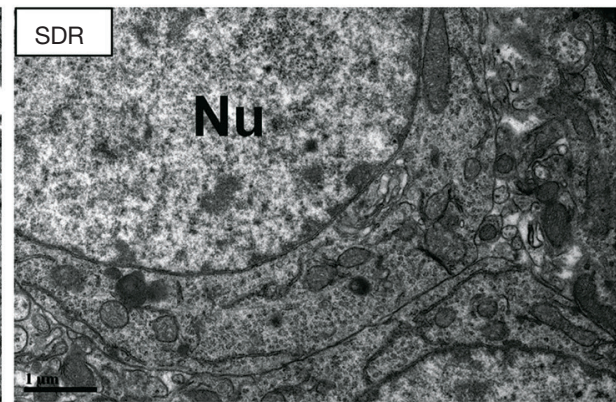

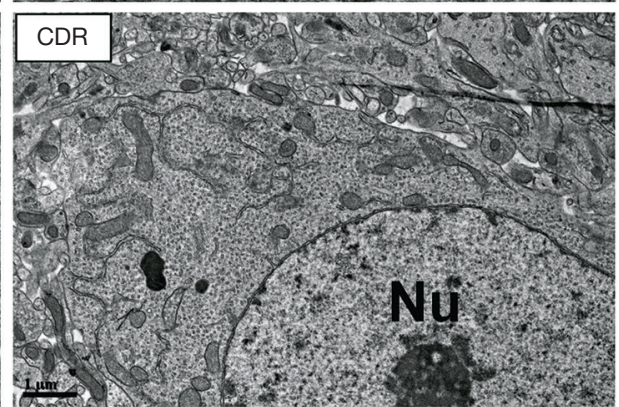

C

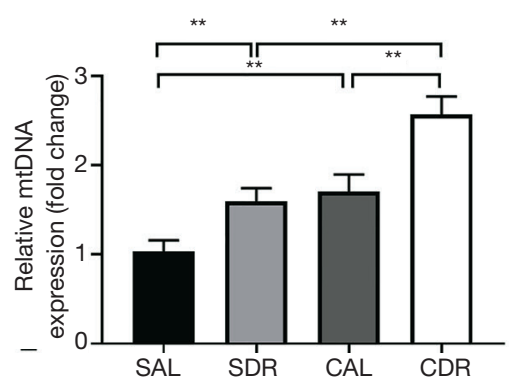

Figure 4 Short-term DR increased brain mitochondrial biogenesis following CA of the rats. (A) Representative transmission electron microscopy (TEM) images of brain mitochondria at $24 \mathrm{~h}$ after CA. The neuronal nucleus $(\mathrm{Nu})$ is surrounded by relatively uniform and compact mitochondria. Scale bar $=1 \mu \mathrm{m}$. (B) Quantification of brain mitochondrial number per photograph by TEM at $24 \mathrm{~h}$ after CA. For each group, $n=3$ and 7 photographs were counted per animal. (C) The brain mitochondrial DNA (mtDNA) copy number was measured using quantitative real-time PCR. DR increased the brain mtDNA copy number at $24 \mathrm{~h}$ after CA. Data in (B) and (C) are shown as mean $\pm \mathrm{SD}, \mathrm{n}=3,{ }^{*}, \mathrm{P}<0.05 ;{ }^{* *}, \mathrm{P}<0.01$; one-way ANOVA followed by Tukey test. SAL, the sham ad libitum diet control group; SDR, the sham dietary restriction control group; CAL, the ad libitum diet and CPR group; CDR, the dietary restriction and CPR group.

recognized. Recent studies demonstrated that DR may enhance brain functions, including learning and memory, synaptic plasticity, and neurogenesis $(31,32)$. The majority of CA survivors have some degree of brain injury in cognitive function due to global brain ischemia. However, whether DR has an effect on neurological outcome after $\mathrm{CA}$ is still unclear. In the present study, we investigated the effect of short-term DR (one week of $70 \%$ reduced daily diet) on neurological injury in rats using an asphyxia CA model. Our results found that brain neurological functions decreased after CA and short-term DR significantly improved 3-day survival and neurological outcome in rats. In addition, analysis of neuronal ischemic injury by Nissl and TUNEL staining revealed that short-term DR significantly attenuated cellular apoptosis and neuronal damage in hippocampal CA1 region. Therefore, these results suggest that short-term DR has a protective effect on neurological injury after CA.

Mitochondrial ROS such as superoxide and peroxide anions, are byproducts of mitochondrial oxidative phosphorylation and cause oxidative damage to proteins, lipids, and DNA. The mtDNA is thought to be particularly 

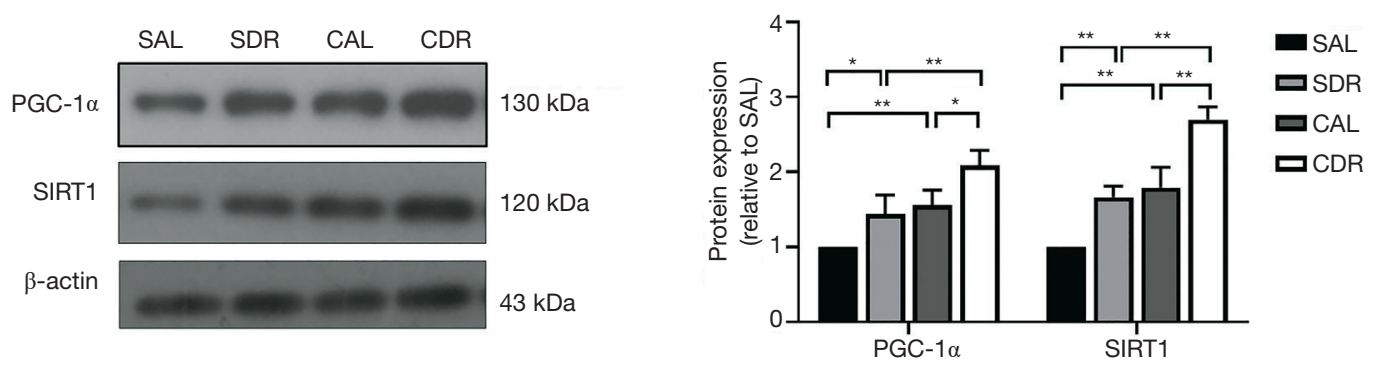

Figure 5 Short-term DR increased brain PGC-1 $\alpha$ and SIRT1 protein expression following CA of the rats. Short-term DR increased brain PGC-1 $\alpha$ and SIRT1 protein expression at $24 \mathrm{~h}$ after CA. Representative results of western blot analysis of PGC-1 $\alpha$ and SIRT1 protein expression in the brain (left). $\beta$-actin was used as the loading control. PGC-1 $\alpha$ and SIRT1 protein expression were quantified by western blot band intensity measurement (right). Data are shown as mean $\pm \mathrm{SD}$. n=3, *, $\mathrm{P}<0.05$; **, $\mathrm{P}<0.01$; one-way ANOVA followed by Tukey test. SAL, the sham ad libitum diet control group; SDR, the sham dietary restriction control group; CAL, the ad libitum diet and CPR group; $\mathrm{CDR}$, the dietary restriction and CPR group.

A

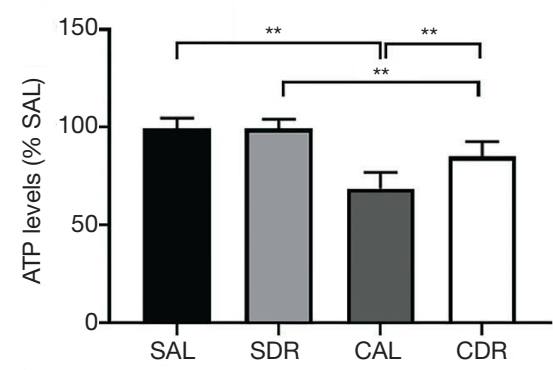

D

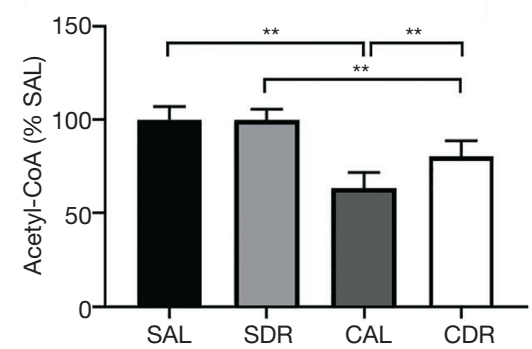

B

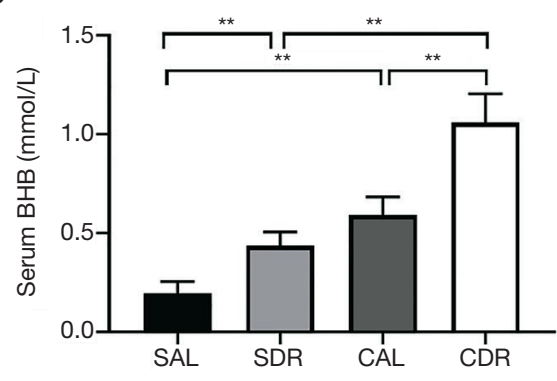

E

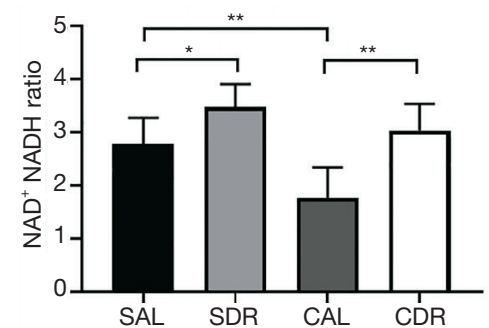

C

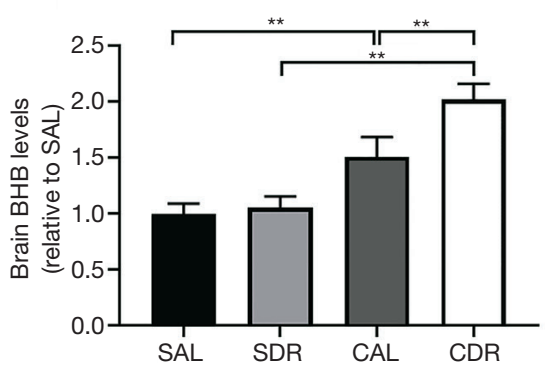

F

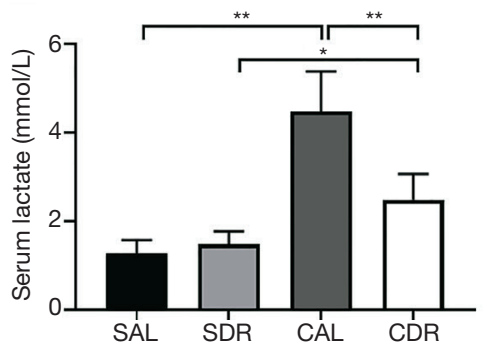

Figure 6 Ketone body involved in short-term DR mediated neurological function protection following CA of the rats. (A) Short-term DR increased the ATP levels of the brain at $6 \mathrm{~h}$ after CA. (B) Short-term DR increased the serum $\beta$-hydroxybutyrate (BHB) levels at $6 \mathrm{~h}$ after CA. (C) Short-term DR increased BHB levels at $6 \mathrm{~h}$ after CA. (D) Short-term DR increased acetyl-CoA levels of the brain at $6 \mathrm{~h}$ after CA. (E) Short-term DR increased $\mathrm{NAD}^{+} / \mathrm{NADH}$ ratio of the brain at $6 \mathrm{~h}$ after $\mathrm{CA}$. (F) Short-term DR decreased the serum lactate levels at $6 \mathrm{~h}$ after CA. Data are shown as mean \pm SD. $\mathrm{n}=6,{ }^{*}, \mathrm{P}<0.05$; ${ }^{* *}, \mathrm{P}<0.01$; one-way ANOVA followed by Tukey test. SAL, the sham ad libitum diet control group; SDR, the sham dietary restriction control group; CAL, the ad libitum diet and CPR group; CDR, the dietary restriction and CPR group. 
susceptible to oxidative damage due to lack of protective histone proteins and its proximity to places of ROS production $(33,34)$. The persistence of mtDNA damage ultimately leads to mutations in the mitochondrial genome and gives rise to further mitochondrial dysfunction, which induces and aggravates the progress of the disease (34). For example, mitochondrial alteration because of oxidative damage and mtDNA mutations leads to degeneration of dopaminergic neurons (35). It has reported that $8-\mathrm{OHdG}$, one of the common oxidative damage markers, was detected at a higher level in mtDNA than nuclear DNA in Alzheimer's disease (36). Consistently, our study showed that brain $8-\mathrm{OHdG}$ staining after CA was primarily around the nuclei, indicating brain mtDNA has more oxidative damages than nuclear DNA after CA. A reduction in mitochondrial ROS production is a widely consistent effect of DR $(20,24)$. Our data demonstrated that shortterm DR significantly decreased brain ROS levels at 6 hours and mitochondrial oxidative damage at 24 hours after CA. Generally, the mechanisms that DR attenuates ROS production are involved in preventing electron leakage from complex I and/or III and increasing antioxidant defense system $(37,38)$.

Mitochondrial biogenesis is a cellular quality control process and is essential for cells and tissues to recover from mitochondrial damage such as ischemic injury. Studies have proven that enhancing mitochondrial biogenesis is an effective strategy to reduce ischemic cerebral injury after CA. For example, exogenous carbon monoxide administration increased mitochondrial ATP production and activity at 24 hours after ROSC in rats via activating mitochondrial biogenesis and therefore decreased brain injury after CA (14). Similar findings also have been reported that sodium hydrosulfide treatment significantly improved neurological outcomes of the rats after ROSC by maintenance of mitochondrial function by improving mitochondrial biogenesis (39). Our data found that shortterm DR increased brain mitochondrial biogenesis after CA by electron microscopy analysis and mtDNA copy number analysis. Therefore, our results suggested that mitochondrial biogenesis may account for short-term DR mediated neuroprotective effect after CA. Mitochondrial biogenesis is regulated by several transcriptional regulators at the transcriptional levels of gene expression. PGC- $1 \alpha$ is a co-transcriptional regulation factor and a master regulator of mitochondrial biogenesis (29). Brain mitochondrial biogenesis in the AL rats was increased after $\mathrm{CA}$ in our results, which can be regulated by AMP-activated protein
kinase/PGC-1 $\alpha$ pathway (40). It has been proved that SIRT1 could activate PGC-1 $\alpha$ and elevate mitochondrial biogenesis $(29,41)$. Our data showed that short-term DR significantly increased the protein levels of SIRT1 and PGC- $1 \alpha$ before and after CA. Therefore, the SIRT1/PGC$1 \alpha$ pathway plays an important role in brain mitochondrial biogenesis under short-term DR.

In our study, we found that the brain ATP levels were decreased and short-term DR increased ATP, BHB and acetyl-CoA levels, as well as $\mathrm{NAD}^{+} / \mathrm{NADH}$ ratios after CA. BHB is the most abundant of the three ketone bodies created by the liver and accounts $78 \%$ of the total ketones in the blood. Ketone bodies are readily transported into other tissues from the liver and converted into acetyl-CoA, which then enters the citric acid cycle and is oxidized in the mitochondria for energy. Based on differential NAD ${ }^{+}$ utilization during glucose or ketone body mediated acetylCoA generation, ketone body metabolism can increase the $\mathrm{NAD}^{+} / \mathrm{NADH}$ ratio (42). Therefore, our results showed that short-term DR increased brain ketone body metabolism for energy supply after CA, which is consistent with the findings that ketone bodies are an alternative fuel for brain cells when glucose availability is insufficient (43). It has reported that acetyl-CoA oxidation from ketone bodies accounted for $62 \%$ of neuronal TCA cycle flux, and the remaining $38 \%$ contributed by glucose in fasted rats (44). Because both brain glucose oxidative metabolism and neuronal activity decline after CA, the ketone body metabolism mediated by short-term DR may be able to sustain brain energy supply and improve brain functions after CA. In this study, we also showed that short-term DR decreased serum lactate levels after CA. The serum lactate level is a good prognostic indicator for tissue perfusion deficit. Elevated lactate concentration has been associated with increased mortality after out-of-hospital CA (45). The blood lactate level is also used as a prognostic predictor for CA patients who undergo therapeutic hypothermia (46). Moreover, evidence showed that ketone body can inhibit glycolysis and thereby decrease lactate production (47). Therefore, our data suggest that ketone bodies are key metabolites for brain energy metabolism and maintain brain function after CA under short-term DR.

We acknowledged that our study had some limitations. First, we only investigated the effect of short-term DR (one week of $70 \%$ reduced daily diet) on brain injury after CA, long-term DR and other levels of reduced daily diet need to further study. Second, our study mainly focused on the early stage of brain injury ( 6 and 24 hours of CA) and the 
associated pathological processes after CA. Future studies should prolong the observation period to assess survival and neurologic outcomes. Finally, we used only adult male rats for all experiments, but CA clinically also occurs in both elderly males and females. Future experiments should investigate short-term DR in aged and female rats to better simulate the human condition.

In conclusion, we found that short-term DR improved survival and neurologic outcomes after CA in rats. Reduction of oxidative stress, upregulation of mitochondrial biogenesis and increase of ketone body metabolism may play a crucial role in preserving neuronal function after CA under short-term DR. However, it remains to further study if the benefits of short-term DR on brain function observed in animals will translate to humans.

\section{Acknowledgments}

Funding: This study was supported by the National Natural Science Foundation of China (No. 81971805; 81501137), Guangdong Basic and Applied Basic Research Foundation (No. 2020A1515010158) and Guangzhou Science and Technology Research Project (No. 201804010061).

\section{Footnote}

Reporting Checklist: The authors have completed the ARRIVE reporting checklist Available at http://dx.doi. org/10.21037/atm-20-3075

Data Sharing Statement: Available at http://dx.doi. org/10.21037/atm-20-3075

Conflicts of Interest: All authors have completed the ICMJE uniform disclosure form (available at http://dx.doi. org/10.21037/atm-20-3075). The authors have no conflicts of interest to declare.

Ethical Statement: The authors are accountable for all aspects of the work in ensuring that questions related to the accuracy or integrity of any part of the work are appropriately investigated and resolved. All animal experiments were carried out in accordance with the National Institutes of Health Guide for the Care and Use of Laboratory Animals (8th edition; Washington DC, National Academic Press, 2011). The protocol was approved by the Institutional Animal Care and Use Committee of Sun Yatsen University (No. IACUC-DB-15-1207).
Open Access Statement: This is an Open Access article distributed in accordance with the Creative Commons Attribution-NonCommercial-NoDerivs 4.0 International License (CC BY-NC-ND 4.0), which permits the noncommercial replication and distribution of the article with the strict proviso that no changes or edits are made and the original work is properly cited (including links to both the formal publication through the relevant DOI and the license). See: https://creativecommons.org/licenses/by-nc-nd/4.0/.

\section{References}

1. Panchal AR, Berg KM, Hirsch KG, et al. 2019 American Heart Association Focused Update on Advanced Cardiovascular Life Support: Use of Advanced Airways, Vasopressors, and Extracorporeal Cardiopulmonary Resuscitation During Cardiac Arrest: An Update to the American Heart Association Guidelines for Cardiopulmonary Resuscitation and Emergency Cardiovascular Care. Circulation 2019;140:e881-e894.

2. Stub D, Bernard S, Duffy SJ, et al. Post cardiac arrest syndrome: a review of therapeutic strategies. Circulation 2011;123:1428-35.

3. Harukuni I, Bhardwaj A. Mechanisms of brain injury after global cerebral ischemia. Neurol Clin 2006;24:1-21.

4. Hypothermia after Cardiac Arrest Study Group. Mild therapeutic hypothermia to improve the neurologic outcome after cardiac arrest. N Engl J Med 2002;346:549-56.

5. Nunnally ME, Jaeschke R, Bellingan GJ, et al. Targeted temperature management in critical care: a report and recommendations from five professional societies. Crit Care Med 2011;39:1113-25.

6. Picard M, Wallace DC, Burelle Y. The rise of mitochondria in medicine. Mitochondrion 2016;30:105-16.

7. Patil KD, Halperin HR, Becker LB. Cardiac arrest: resuscitation and reperfusion. Circ Res 2015;116:2041-9.

8. Donnino MW, Liu X, Andersen LW, et al. Characterization of mitochondrial injury after cardiac arrest (COMICA). Resuscitation 2017;113:56-62.

9. Kilbaugh TJ, Sutton RM, Karlsson M, et al. Persistently Altered Brain Mitochondrial Bioenergetics After Apparently Successful Resuscitation From Cardiac Arrest. J Am Heart Assoc 2015;4:e002232.

10. Sims NR, Muyderman H. Mitochondria, oxidative metabolism and cell death in stroke. Biochim Biophys Acta 2010;1802:80-91.

11. Liu J, Wang Y, Zhuang Q, et al. Protective effects of 
cyclosporine A and hypothermia on neuronal mitochondria in a rat asphyxial cardiac arrest model. Am J Emerg Med 2016;34:1080-5.

12. Ikeda K, Liu X, Kida K, et al. Thiamine as a neuroprotective agent after cardiac arrest. Resuscitation 2016;105:138-44.

13. Grieb P, Ryba MS, Debicki GS, et al. Changes in oxidative stress in the rat brain during post-cardiac arrest reperfusion, and the effect of treatment with the free radical scavenger idebenone. Resuscitation 1998;39:107-13.

14. Wang P, Yao L, Zhou LL, et al. Carbon Monoxide Improves Neurologic Outcomes by Mitochondrial Biogenesis after Global Cerebral Ischemia Induced by Cardiac Arrest in Rats. Int J Biol Sci 2016;12:1000-9.

15. Gong P, Hua R, Zhang Y, et al. Hypothermia-induced neuroprotection is associated with reduced mitochondrial membrane permeability in a swine model of cardiac arrest. J Cereb Blood Flow Metab 2013;33:928-34.

16. Hackenhaar FS, Medeiros TM, Heemann FM, et al. Therapeutic Hypothermia Reduces Oxidative Damage and Alters Antioxidant Defenses after Cardiac Arrest. Oxid Med Cell Longev 2017;2017:8704352.

17. Wang P, Li Y, Yang Z, et al. Inhibition of dynamin-related protein 1 has neuroprotective effect comparable with therapeutic hypothermia in a rat model of cardiac arrest. Transl Res 2018;194:68-78.

18. Thoresen M, Penrice J, Lorek A, et al. Mild hypothermia after severe transient hypoxia-ischemia ameliorates delayed cerebral energy failure in the newborn piglet. Pediatr Res 1995;37:667-70.

19. Robertson LT, Mitchell JR. Benefits of short-term dietary restriction in mammals. Exp Gerontol 2013;48:1043-8.

20. Amigo I, Kowaltowski AJ. Dietary restriction in cerebral bioenergetics and redox state. Redox Biol 2014;2:296-304.

21. Witte AV, Fobker M, Gellner R, et al. Caloric restriction improves memory in elderly humans. Proc Natl Acad Sci U S A 2009;106:1255-60.

22. Chandrasekar B, Nelson JF, Colston JT, et al. Calorie restriction attenuates inflammatory responses to myocardial ischemia-reperfusion injury. Am J Physiol Heart Circ Physiol 2001;280:H2094-102.

23. Yu ZF, Mattson MP. Dietary restriction and 2-deoxyglucose administration reduce focal ischemic brain damage and improve behavioral outcome: evidence for a preconditioning mechanism. J Neurosci Res 1999;57:830-9.

24. Pani G. Neuroprotective effects of dietary restriction:
Evidence and mechanisms. Semin Cell Dev Biol 2015;40:106-14.

25. Martin-Montalvo A, de Cabo R. Mitochondrial metabolic reprogramming induced by calorie restriction. Antioxid Redox Signal 2013;19:310-20.

26. Qin J, Wang P, Li Y, et al. Activation of Sigma-1 Receptor by Cutamesine Attenuates Neuronal Apoptosis by Inhibiting Endoplasmic Reticulum Stress and Mitochondrial Dysfunction in a Rat Model of Asphyxia Cardiac Arrest. Shock 2019;51:105-13.

27. Geocadin RG, Ghodadra R, Kimura T, et al. A novel quantitative EEG injury measure of global cerebral ischemia. Clin Neurophysiol 2000;111:1779-87.

28. Wang P, Sun YC, Lu WH, et al. Selective killing of K-rastransformed pancreatic cancer cells by targeting $\mathrm{NAD}(\mathrm{P})$ H oxidase. Chin J Cancer 2015;34:166-76.

29. Fernandez-Marcos PJ, Auwerx J. Regulation of PGC1alpha, a nodal regulator of mitochondrial biogenesis. Am J Clin Nutr 2011;93:884S-90.

30. Stein LR, Imai S. The dynamic regulation of NAD metabolism in mitochondria. Trends Endocrinol Metab 2012;23:420-8.

31. Fontán-Lozano A, Lopez-Lluch G, Delgado-Garcia JM, et al. Molecular bases of caloric restriction regulation of neuronal synaptic plasticity. Mol Neurobiol 2008;38:167-77.

32. Qiu G, Liu S, So KF. Dietary restriction and brain health. Neurosci Bull 2010;26:55-65.

33. Santos RX, Correia SC, Zhu X, et al. Mitochondrial DNA oxidative damage and repair in aging and Alzheimer's disease. Antioxid Redox Signal 2013;18:2444-57.

34. Cha MY, Kim DK, Mook-Jung I. The role of mitochondrial DNA mutation on neurodegenerative diseases. Exp Mol Med 2015;47:e150.

35. Jin H, Kanthasamy A, Ghosh A, et al. Mitochondriatargeted antioxidants for treatment of Parkinson's disease: preclinical and clinical outcomes. Biochim Biophys Acta 2014;1842:1282-94.

36. Wang J, Xiong S, Xie C, et al. Increased oxidative damage in nuclear and mitochondrial DNA in Alzheimer's disease. J Neurochem 2005;93:953-62.

37. Qiu X, Brown K, Hirschey MD, et al. Calorie restriction reduces oxidative stress by SIRT3-mediated SOD2 activation. Cell Metab 2010;12:662-7.

38. Shinmura K, Tamaki K, Sano M, et al. Caloric restriction primes mitochondria for ischemic stress by deacetylating specific mitochondrial proteins of the electron transport chain. Circ Res 2011;109:396-406. 


\section{Page 14 of 14}

39. Pan H, Xie X, Chen D, et al. Protective and biogenesis effects of sodium hydrosulfide on brain mitochondria after cardiac arrest and resuscitation. Eur J Pharmacol 2014;741:74-82.

40. Jiang S, Li T, Ji T, et al. AMPK: Potential Therapeutic Target for Ischemic Stroke. Theranostics 2018;8:4535-51.

41. Nemoto S, Fergusson MM, Finkel T. SIRT1 functionally interacts with the metabolic regulator and transcriptional coactivator PGC-1\{alpha\}. J Biol Chem 2005;280:16456-60.

42. Elamin M, Ruskin DN, Masino SA, et al. Ketone-Based Metabolic Therapy: Is Increased $\mathrm{NAD}(+)$ a Primary Mechanism? Front Mol Neurosci 2017;10:377.

43. Zhang Y, Kuang Y, Xu K, et al. Ketosis proportionately spares glucose utilization in brain. J Cereb Blood Flow Metab 2013;33:1307-11.

Cite this article as: Zhou M, Yu T, Fang X, Ge Q, Song F, Huang Z, Jiang L, Wang P. Short-term dietary restriction ameliorates brain injury after cardiac arrest by modulation of mitochondrial biogenesis and energy metabolism in rats. Ann Transl Med 2021;9(1):8. doi: 10.21037/atm-20-3075

\section{Zhou et al. Short-term DR improves neurological outcomes}

44. Chowdhury GM, Jiang L, Rothman DL, et al. The contribution of ketone bodies to basal and activitydependent neuronal oxidation in vivo. J Cereb Blood Flow Metab 2014;34:1233-42.

45. Shinozaki K, Oda S, Sadahiro T, et al. Blood ammonia and lactate levels on hospital arrival as a predictive biomarker in patients with out-of-hospital cardiac arrest. Resuscitation 2011;82:404-9.

46. Lee DH, Cho IS, Lee SH, et al. Correlation between initial serum levels of lactate after return of spontaneous circulation and survival and neurological outcomes in patients who undergo therapeutic hypothermia after cardiac arrest. Resuscitation 2015;88:143-9.

47. Melø TM, Nehlig A, Sonnewald U. Neuronal-glial interactions in rats fed a ketogenic diet. Neurochem Int 2006;48:498-507. 